\title{
Efficacy of IV Immunoglobulins on Psychiatric Symptoms: A Case Report
}

\author{
Silva A $^{1}$, M Gastaldi ${ }^{2,3}$, C Placenti ${ }^{1}$, A Donadeo ${ }^{1}$, P Politi ${ }^{1}$, N Brondino ${ }^{1}$ and M Olivola ${ }^{1 *}$ \\ ${ }^{1}$ Department of Brain and Behavioral Sciences, University of Pavia, Italy \\ ${ }^{2}$ IRCCS, C. Mondino National Neurological Institute, Pavia, Italy \\ ${ }^{3}$ University of Pavia, Pavia, Italy
}

*Corresponding author: Miriam Olivola, consultant psychiatrist Servizio Psichiatrico di Diagnosi e Cura ASST Pavia, Viale

Repubblica 34 - 27100 Pavia presso IRCCS Policlinico San Matteo di Pavia, Italy

\begin{tabular}{lll}
\hline ARTICLE INFO & & ABSTRACT \\
\cline { 1 - 1 } Received: & & Abbreviations: IVIG: Intravenous Immunoglobulins; MDD: Major Depressive Disorder; \\
Published: 2021 & & BPD: Borderline Personality Disorder
\end{tabular}

Citation: Silva A, M Gastaldi, C Placenti, A Donadeo, M Olivola, et al., Efficacy of IV Immunoglobulins on Psychiatric Symptoms: A Case Report. Biomed J Sci \& Tech Res 37(5)-2021. BJSTR. MS.ID.006057.

\section{Introduction}

Borderline personality disorder (BPD) is a fairly common psychiatric illness with a prevalence between $1.8 \%$ and $2 \%$ in the general population [1]. Diagnostic Criteria in the DSM-5 [2]: BPD is a pervasive pattern of instability in interpersonal relationships, self-image, and mood and marked impulsivity that begins by early adulthood and is present in a variety of contexts, as indicated by five (or more) of the following:

a) Desperate efforts to avoid real or imagined abandonment (do not include behaviours indicated in criterion 5). A pattern of unstable and intense interpersonal relationships, characterized by the alternating between extremes of hyper-idealisation and devaluation.

b) Altered identity: markedly and persistently unstable selfimage or self-perception.

c) Impulsivity in at least two areas that are potentially harmful to the subject (e.g. reckless spending, promiscuous sex, substance abuse, reckless driving, binge drinking,). d) Recurrent suicidal behaviour, gestures or threats, or self-mutilating behaviour (self-harm, cuts on arms and legs, cigarette burns, etc.).

e) Affective instability due to marked mood reactivity (e.g., episodic intense dysphoria, irritability or anxiety, usually lasting a few hours, and only rarely more than a few days).

f) Chronic feelings of emptiness.

g) Inappropriate, intense anger or difficulty controlling anger (e.g., frequent bouts of anger or constant rage, recurrent physical confrontations).

h) Transient paranoid ideation, associated with stress, or severe dissociative symptoms.

Individuals with this disorder have recurrent suicidal behavior, gestures or threats, or self-harming behavior. Successful suicide occurs in $8-10 \%$ of such individuals, and self-harming gestures (e.g., cutting or burning oneself) and suicide threats and attempts are very common (DSM-5) [2]. Major depressive disorder (MDD) is the most 
frequent comorbidity with a prevalence of 41-83\% [3], followed by substance use disorder with a prevalence of 78\% [4]. The basic therapy of BPD is psychotherapy while psychopharmacology has an additional role as symptomatic and treating comorbidities [5]. Current evidence from randomised controlled trials suggests that pharmacological treatment, particularly with mood stabilisers and second-generation antipsychotics, may be effective for symptomatic treatment, but the evidence does not currently support efficacy for the overall severity of BPD [6].

Some studies in literature highlight the correlation between immune-mediated inflammation and psychiatric disease. Intravenous immunoglobulins (IVIG) are a widely used therapy in the field of autoimmune diseases, and in particular in several neuroimmunological disorders [7]. IVIGs regulate the immune system with multiple mechanisms: modulation of $\mathrm{Fc}$ receptor function, interference with cytokine release and function; interference with complement activation; presence of anti-idiotype antibodies; modulation of B and T lymphocyte activity. There are few studies about the efficacy of IV immunoglobulins in psychiatric features [8-10].

\section{Case Presentation}

A 40-year patient diagnosed with borderline personality disorder and major depressive disorder in comorbidity with myasthenia was hospitalized for self-harm and suicidal behavior due to impulsivity. She showed particularly pronounced levels of the self-conscious emotions, shame and guilt, instability of affect, emotion dysregulation, feeling of emptiness, lack of interest to all activity, sleep disturbance, anxiety and depressed mood. Pharmacological treatment with acid valproic, aripiprazole and gabapentin were prescribed in addition to pyridostigmine. In the psychiatric history she was diagnosed with drug addiction (quetiapine, benzodiazepine, sedative antipsychotics and cocaine) and eating disorder. During hospitalization she had daily non suicidal self-injuries, agitation and she showed craving for quetiapine, benzodiazepine, sedative antipsychotics. After one week of hospitalization, she had a myasthenic crisis, glucocorticoids were contraindicated due to the psychiatric side effects, so intravenous immunoglobulins (IVIG) were prescribed by neurologist $(0.4 \mathrm{mg} /$ $\mathrm{kg}$ /day for 5 days) in addition to azathioprine.

After one day of IVIG the psychiatric features improved: patient reported an improved mood, lower level of agitation, a reduction of self-harm behaviors and an improved nighttime sleep. After sixth days of IVIG was observed a remission of myasthenic crisis by the neurologist and the IVIG were stopped. Consolidated the neurological and psychiatric improvements, the patient was discharged. After 8 months, at the follow-up visit, the patient had no re-exacerbations of psychiatric or myasthenic symptoms, she regularly took the prescribed therapy with acid valproic, aripiprazole, gabapentin, pyridostigmine and azathioprine and she also has been in psychotherapy for 7 months and she has started a new job in addition to taking care of her two children. At the admission in hospital (T0), an assessment was performed by the clinician with the following interviews:

a) Inventory of Statements About Self-Injury (ISAS)

b) Barrat Impulsiveness Scale, Version 11 (BIS-11)

c) Hamilton Anxiety Rating Scale (HAM-A)

d) Montgomery-Asberg Depression Rating Scale (MADRS)

e) Alexian Brothers Urge to Self-Injure Scale (ABUSI)

The same evaluation was repeated at the end of the IgEV administration ( 6 days - T1) and after 8 months (T2) without other treatment modifications.

\section{Results}

The patient presented a score of 79 at BIS-11. She reported a huge number of self-harm and suicidal behaviors as reported on ISAS (Figure 1). In Figure 2 we can see the reduction of anxiety, depressive symptoms and self-harm from to to $\mathrm{t} 2$.

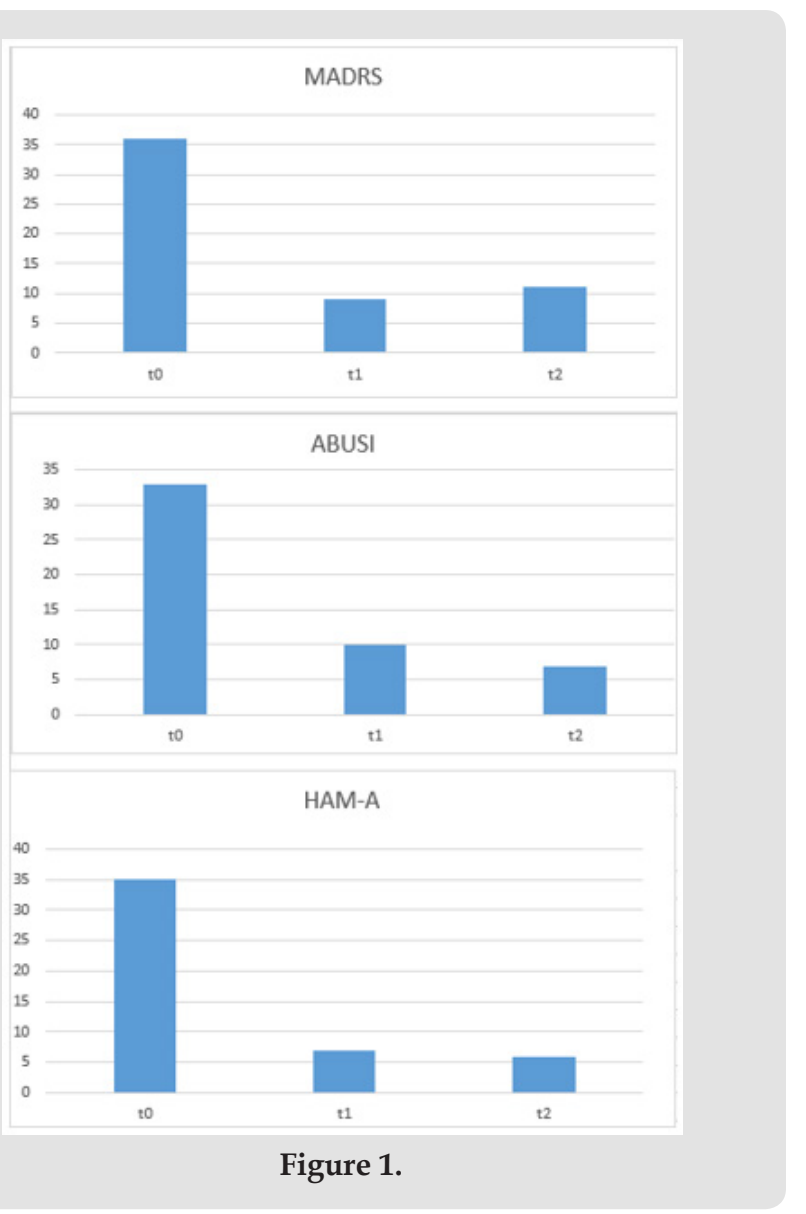




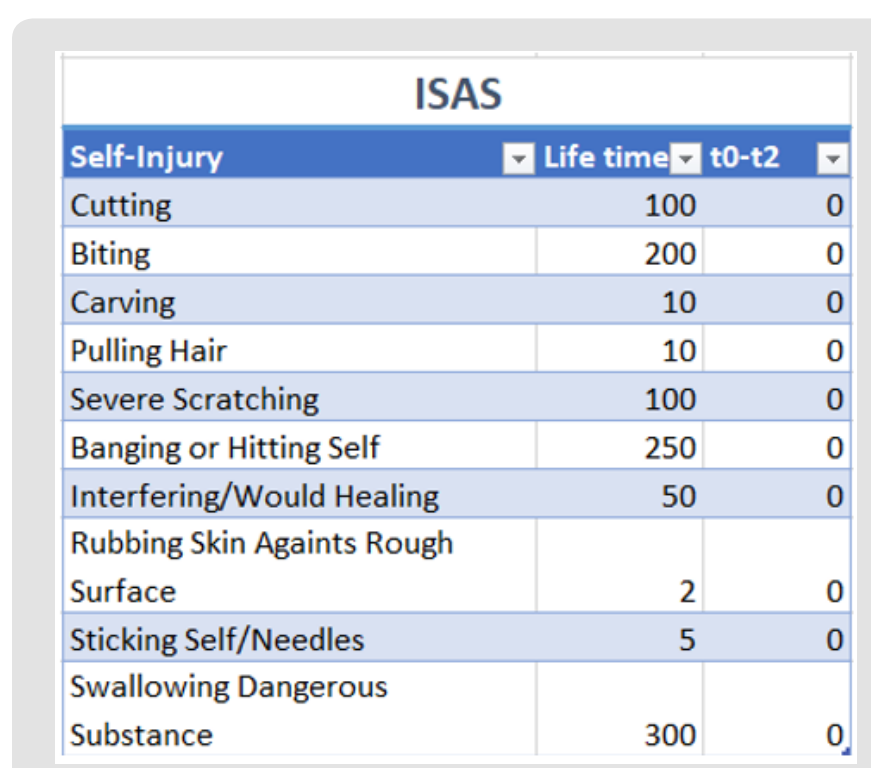

Figure 2.

\section{Conclusion}

We observed a reduction in self harm, impulsivity and a remission of depressive symptoms, only mild level of anxiety was reported immediately after the administration of immunoglobulins, and the remission continued up to 8 months after the administration (Figure 2). Further studies are needed to explain the mechanism that led to remission of psychiatric symptoms after administration of IVIG. Even though, the remission of the myasthenic crisis has led to an improvement of the psychiatric features, further causes cannot be excluded. Some studies in literature highlight the correlation between immune-mediated inflammation and

\section{ISSN: 2574-1241}

DOI: 10.26717/BJSTR.2021.37.006058

M Olivola. Biomed J Sci \& Tech Res

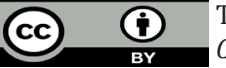

This work is licensed under Creative Commons Attribution 4.0 License

Submission Link: https://biomedres.us/submit-manuscript.php psychiatric disease. However, there are few studies about the efficacy of IV immunoglobulins in psychiatric features which will needed to have more evidence and to better understanding the mechanism of action [8-10].

\section{References}

1. Widiger TA, Weissman MM (1991) Epidemiology of borderline personality disorder. Psychiatric Services 42(10): 1015-1021.

2. (2015) American Psychiatric Association. Neurodevelopmental Disorders: DSM- ${ }^{\oplus}$ Selections. American Psychiatric Pub.

3. Lieb K, Zanarini MC, Schmahl C, Linehan MM, Bohus M (2004) Borderline personality disorder. The Lancet 364(9432): 453-461.

4. Kienast T, Stoffers J, Bermpohl F, Lieb K (2014) Borderline personality disorder and comorbid addiction: epidemiology and treatment. Deutsches Ärzteblatt International 111(16): 280-286.

5. Gabbard GO (2014) Gabbard's Treatments of Psychiatric Disorders, In: MD Glen 0 Gabbard (Edt.).,

6. Lieb K, Völlm B, Rücker G, Timmer A, Stoffers JM (2010) Pharmacotherapy for borderline personality disorder: Cochrane systematic review of randomised trials. The British Journal of Psychiatry 196(1): 4-12.

7. Bayary J, Dasgupta S, Misra N, Ephrem A, Van Huyen, et al. (2006). Intravenous immunoglobulin in autoimmune disorders: an insight into the immunoregulatory mechanisms. International immunopharmacology 6(4): 528-534.

8. Zunszain Patricia A, Hepgul Nilay, Pariante Carmine M (2013) Inflammation and depression. In: Behavioral neurobiology of depression and its treatment. Springer, Berlin, Heidelberg, pp. 135-151.

9. Díaz Marsá M, MacDowell KS, Guemes I, Rubio V, Carrasco JL, et al. (2012) Activation of the cholinergic anti-inflammatory system in peripheral blood mononuclear cells from patients with borderline personality disorder. Journal of psychiatric research 46(12): 1610-1617.

10. MacDowell KS, Marsá MD, Buenache E, Villatoro JML, Moreno B, et al. (2020) Inflammatory and antioxidant pathway dysfunction in borderline personality disorder. Psychiatry research 284: 112782.

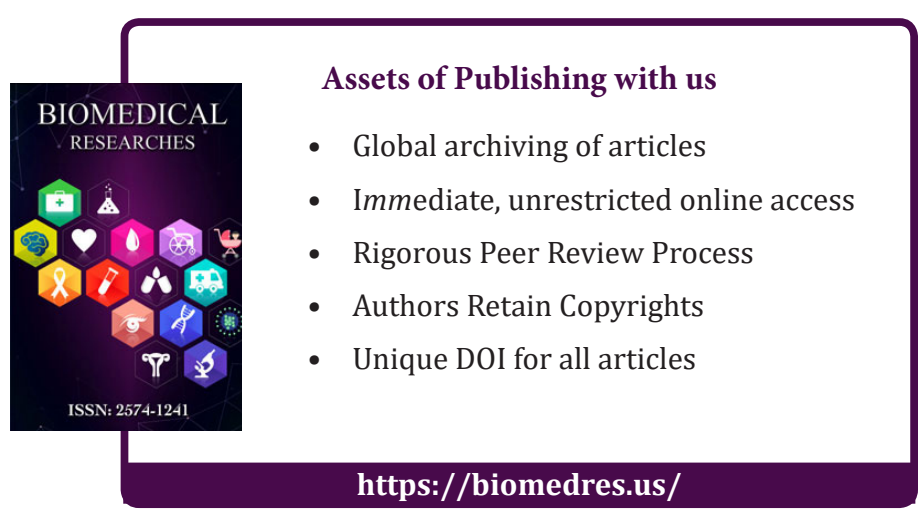

